\title{
EDITORIAL
}

\section{WMS Practice Guidelines Development: Commentary from the Co-Chairs of the WMS Practice Guidelines Committee}

This edition of Wilderness and Environmental Medicine (WEM) contains the eighth installment of the Wilderness Medical Society (WMS) practice guidelines, WMS Practice Guidelines for the Prevention and Treatment of Heat Related Illness. As the WMS continues to develop clinical practice guidelines (CPGs), we believe this is an opportune time to communicate to our members and readers the process the Society has adopted for the development and implementation of CPGs.

The original WMS Practice Guidelines for Wilderness Emergency Care were published in text form in 1980, with five subsequent editions, most recently in 2005. Although these guidelines have provided a valuable source of information for our membership and beyond, the WMS leadership believes that current and future guidelines should be published in a more contemporary format subject to a more scientifically rigorous and transparent process as outlined below.

CPGs have become ubiquitous as the medical literature database has increased exponentially over the past two decades. The explosion in medical literature has created a voluminous amount of information impossible for the average clinician to navigate. As a result, a parallel explosion of CPGs has occurred. Unfortunately, however, a unified and reliable approach to the development of CPGs has not yet been universally adopted. The reasons for this are multi-factorial, however one major contributing factor is the wide difference in available evidence across different specialties and medical conditions of widely varying incidence. Well designed and adequately powered randomized clinical trials (RCTs) lend themselves well to situations where common ailments (such as diabetes mellitus or hypertension) can be treated dichotomously (medication vs. placebo). This is particularly true when blinding can be easily accomplished. High level evidence is more difficult to obtain in procedural specialties (subject to large variations in technique, myriad related biases, and difficulty blinding) and rare conditions. Certainly, the latter is a challenge in austere medicine. The illnesses and injuries we treat are not only rare, but occur in challenging and remote environments. It is unlikely that we will ever amass high level evidence in the treatment of high altitude cerebral edema, arthropod bites, or lightning strikes. Even more common conditions such as frostbite, acute mountain sickness, hypothermia, and submersion are relatively infrequent, disparate, and unpredictable.

In 2008 the Institute of Medicine (IOM) published guidelines for the development of a standardized approach for objective, scientifically valid, and consistent approaches to developing CPGs. ${ }^{1}$ In spite of these efforts, an independent review of 130 guidelines subsequently published, revealed that less than half of these CPGs met more than $50 \%$ of the IOM standards. ${ }^{2}$

The following represents the WMS process to develop trustworthy CPGs, based on the principles outlined by the IOM:

Transparency. The WMS will clearly publicize the process by which CPGs are developed. This communication is one example of an effort to do so. The CPG Committee meetings are open to all WMS members.

Guidelines Development Group Composition. Guideline panel membership is a main determinant of the trustworthiness of guidelines. ${ }^{3}$ Most panels are composed of authors representing a single specialty and therefore sharing similar values and biases. The WMS is committed to insuring that each CPG is developed by a working group not only representative of the best experts in the field but truly multi-disciplinary in scope. The heat illness CPG in this issue of WEM represents that commitment with representation from emergency, family, and pulmonary/critical care medicine as panel members.

Conflicts of Interest. The WMS CPG development protocol requires complete disclosure of conflicts of interest of all panel members as the first order of business once a panel is formed. Panel members with significant conflicts of interest must be removed from the panel and cannot be an author. Conflicted panel members may serve in an advisory capacity only.

Use of Best Evidence Available. The WMS is committed to ensuring that each CPG panel, as a whole, possesses a sufficient level of expertise to search, identify, critique, and grade the available evidence to insure the most inclusive search and identification of the 
highest level of evidence. There remains a dearth of RCTs or even good prospective data collection for many of the problems faced by wilderness medicine providers; when necessary, we have been candid about the use of consensus expert opinion in lieu of solid evidence.

Evidence Grading/Classification. Among several strong guideline systems currently available, this committee believes the classification scheme developed by the American College of Chest Physicians ${ }^{4}$ (ACCP) best fits the WMS CPGs. As discussed above, some of our guidelines will be based on minimal or low-quality evidence. Many of our recommendations will be graded as strong or weak based more on the benefit vs. risk equation than the quality of evidence. Among the available guideline systems, the ACCP classification system offers the greatest ability to assign grades with a clear delineation of how the strength of the recommendation is influenced by both the evidence and the risk/benefit ratio. Other systems are less clear in this regard, or seemingly equate the strength of the recommendation disproportionately to the strength of evidence. While this approach is no doubt appropriate in clinical areas where high levels of evidence exist, we believe it is less transparent in those areas where the preponderance of evidence is of lower quality.

Evidence Quality and Recommendation Strength. For each recommendation, the CPGs should provide sound reasoning, satisfactory discussion of benefits and risks, and describe the relative strength of the evidence and the role of subjective judgments. If there are differences of opinion amongst panel members, these differences should be noted and discussed. Relevant evidence should be easy for the reader to identify for further reference.

Articulating Recommendations. CPGs should clearly articulate the action recommended and the circumstances around which the action should be taken.

External Review. All WMS CPGs are submitted to $W E M$ and are subjected to the same rigorous peer review process, including outside expert review, as any other submitted manuscript. Each guideline is then reviewed and endorsed by the Executive Board of the WMS prior to publication.

Updating. The WMS is committed to timely updating and revising of all CPGs when new or relevant evidence becomes available.

We believe the process as outlined above represents the best attempt of the Society to present CPGs that are both clinically valuable, transparent and trustworthy. Additionally, we are committed to communicating to as many "end-users" as possible to solicit feedback on the utility and effectiveness of CPG implementation and will use this data to influence subsequent updated versions of the guidelines. The previously published PGs have been some of the most widely read and highly cited articles in WEM, and we are both honored and humbled by the task of providing further guidance to our readers through future practice guidelines.

Tracy Cushing, MD, MPH

Robert Quinn, MD

Co-Chairs, Wilderness Medical Society Practice Guidelines Committee

\section{References}

1. IOM (Institute of Medicine). Clinical Practice Guidelines We Can Trust. Washington, DC: National Academies Press; 2011.

2. Kung J, Miller RR, Mackowiak PA. Failure of clinical practice guidelines to meet the Institute of Medicine standards: two decades of little, if any, progress. Arch Intern Med. 2012;172:1628-1633.

3. Shaneyfelt T. In guidelines we cannot trust. Arch Intern Med. 2012;172:1633-1644.

4. Guyatt G, Gutterman D, Baumann M, et al. Grading strength of recommendations and quality of evidence in clinical guidelines. CHEST. 2006;129:174-181. 